\title{
Pioneer anomaly: What can we learn from LISA?
}

\author{
Denis Defrère* $\quad$ Andreas Rathke ${ }^{\dagger}$
}

\begin{abstract}
The Doppler tracking data from two deep-space spacecraft, Pioneer 10 and 11, show an anomalous blueshift, which has been dubbed the "Pioneer anomaly". The effect is most commonly interpreted as a real deceleration of the spacecraft - an interpretation that faces serious challenges from planetary ephemerides. The Pioneer anomaly could as well indicate an unknown effect on the radio signal itself. Several authors have made suggestions how such a blueshift could be related to cosmology. We consider this interpretation of the Pioneer anomaly and study the impact of an anomalous blueshift on the Laser Interferometer Space Antenna (LISA), a planned joint ESA-NASA mission aiming at the detection of gravitational waves. The relative frequency shift (proportional to the light travel time) for the LISA arm length is estimated to $10^{-16}$, which is much bigger than the expected amplitude of gravitational waves. The anomalous blueshift enters the LISA signal in two ways, as a small term folded with the gravitational wave signal, and as larger term at low frequencies. A detail analysis shows that both contributions remain undetectable and do not impair the gravitational-wave detection. This suggests that the Pioneer anomaly will have to be tested in the outer Solar system regardless if the effect is caused by an anomalous blueshift or by a real force.
\end{abstract}

\section{Introduction}

The Laser Interferometer Space Antenna (LISA) is a joint ESA-NASA mission to be launched after 2012 that will detect gravitational waves (GWs) in a frequency range between $10^{-4}$ and $1 \mathrm{~Hz}$ and study their sources [1]. LISA will consist of three spacecraft forming a roughly equilateral triangle of $5 \times 10^{9} \mathrm{~m}$ baseline placed on an orbit similar to that of the Earth. The spacecraft will exchange phase-coherent laser signals with each other in order to conduct picometer interferometry to measure passing GWs through the modulation in the light travel time between the spacecraft that the waves cause.

In this study we consider the impact of an anomalous blueshift, which is homogeneous in the light travel time and isotropic, on LISA. The motivation to consider such an effect comes from the Doppler tracking data of the Pioneer 10 and 11 deep space probes. Both spacecraft show a deviation between their orbit reconstruction and their Doppler tracking signal [2, 3]. The discrepancy, that has become known as the Pioneer anomaly, can correspond either to a small constant deceleration of the spacecraft of roughly $9 \times 10^{-10} \mathrm{~m} / \mathrm{s}^{2}$ or to an anomalous blueshift of the radio signal at a rate of $6 \times 10^{-9} \mathrm{~Hz} / \mathrm{s}$. Since no unambiguous conventional mechanism, like small on-board forces, to explain the anomaly has been identified there is a growing number of studies, which consider an explanation in terms of a novel physical effect (see [3, 4, 5] for an overview of the theoretical models). It has been realised that it is difficult to explain the Pioneer anomaly by a real force which satisfies all constraints from planetary ephemerides 3. 5. 6. Hence an explanation in terms of an anomalous blueshift seems particularly attractive.

In view of the increasing interest in an experimental verification of the Pioneer anomaly [7] it is a logical step to consider if such a verification might be possible with a space mission that is already planned. Unfortunately, the current and upcoming exploration missions are hardly suited for a verification of the Pioneer anomaly [5, 8]. LISA is the first upcoming high-precision fundamental-physics mission that might

*Faculty of Applied Sciences, University of Liege, chemin des Chevreuils, 1 Bât. B52/3 Sart Tilman, 4000 Liege, Belgium, email: denis.defrere@skynet.be

${ }^{\dagger}$ Institute for Theoretical Physics, University of Cologne, Zuelpicher Str. 77, 50937 Cologne, Germany, email: ra@thp.unikoeln.de 
be sensitive to the anomaly. Already in an early discussion, L. Scheffer expressed the expectation, that the Pioneer anomaly, if not due to a spacecraft-specific conventional reason, should be detectable in data from LISA [9]. In a proposal to ESA's 'Cosmic Vision 2015-2025 Call for Themes' the question was raised again if LISA could be a suitable testbed for a verification of the Pioneer anomaly - in particular if the effect were due to an anomalous blueshift [10]. Even more important might be the opposite question: If the Pioneer anomaly is indeed a novel physical effect could it impair the performance of LISA? In this case it would be of crucial importance to ensure that the LISA science goals can be achieved despite of the presence of the anomaly. The present study addresses both of these questions and comes to the conclusion that LISA is neither sufficiently sensitive to the Pioneer anomaly to detect it nor impeded in its mission goals by the potential presence of the anomaly.

The layout of our considerations is as follows. Section 2 gives an overview of the Pioneer anomaly and its possible relevance for LISA. In Section 2.1 we review the observational evidence for the Pioneer

anomaly, and briefly review the models, that have been put forward to explain the anomaly. In Section 2.2 we discuss which models of the Pioneer anomaly are relevant for observations with LISA and derive a first order of magnitude estimate for the maximal effect to expect on the interferometric signal of LISA. We also find the generic response function of LISA in the presence of an anomalous blueshift. Section 3 discusses the effect of the blueshift in the frequency domain. The frequency domain method has been discarded for the actual evaluation of LISA interferometric data because time-delay interferometry (TDI) achieves a far superior cancellation of the laser phase noise (cf. [11). However the frequency domain method has the advantage that it gives direct physical insight into the impact of an anomalous blueshift on the interferometer. Section 3.1 briefly reviews the structure of the interferometric signal of LISA and its Fourier transform. It is followed by the analysis of the impact of the blueshift, which is split into two parts. First Section 3.2 discusses the effect of the anomalous blueshift in the sensitivity band of LISA. Then the detectability of the blueshift at very low frequencies outside of the sensitivity band of LISA is considered in Section 3.3. In both cases no measurable impact of the anomaly is found. Section 4 reconsiders the effect of the anomaly in the framework of TDI, the current baseline method for LISA. Section 4.1 discusses the signature of the anomaly on first generation TDI observables for the idealised case of fixed arm length. It is found that the symmetry of TDI observables leads to an exact cancellation of the effect of the anomalous blueshift in the case of fixed interferometer arms. Section 4.2 generalises these considerations to the realistic case of moving spacecraft. Also in this setting the effect of the anomalous blueshift would remain below the detection threshold of LISA. Section 5 summarises our results and discusses their implications for options to verify and characterise the Pioneer anomaly.

\section{The Pioneer anomaly and LISA}

\subsection{The characteristics of the Pioneer anomaly}

The Pioneer 10 and 11 spacecraft, launched on 2 March 1972 and 5 April 1973, respectively, were the first to explore the outer Solar system (see [12] for an overview of the Pioneer 10 and 11 missions.). Since its Jupiter gravity assist on 4 December 1973 Pioneer 10 is on a hyperbolic coast. Pioneer 11 used a Saturn swingby on 1 September 1979 to reach a hyperbola, in approximately opposite direction to Pioneer 10. Already before the swingby a discrepancy between the Doppler signal from Pioneer 10 and its orbit integration was observed, which was originally ascribed to fuel leaks and a mismodelling in the Solar radiation pressure model (cf. [13]). This interpretation became more and more untenable after the swingbys due to the decrease of the Solar radiation pressure, inversely proportional to the square of the heliocentric distance, and the quiet state of the spacecraft, with very little thruster activity. Moreover an anomaly of the same magnitude became apparent in the Pioneer 11 data 14 .

The anomaly on both probes has been subject to three independent analyses with different orbit determination programs [3, 15]. The result of the investigations is that an anomalous Doppler blueshift is present in the data from both spacecraft of approximately $6 \times 10^{-9} \mathrm{~Hz} / \mathrm{s}$ corresponding to an apparent deceleration of the spacecraft of approximately $9 \times 10^{-10} \mathrm{~m} / \mathrm{s}^{2}$. From the Doppler data, it is not possible to 
distinguish between an anomalous frequency shift of the radio signal or a real deceleration of the spacecraft (see below). The principle investigators of the anomaly have conducted a thorough investigation of possible biases and concluded that no conventional effect is likely to have caused the anomaly [3]. Meanwhile, there exists an ample body of literature discussing various aspects of possible systematic effects, without definitive conclusion 9, 16, 17, 18, 19, 20]. For various reasons all other deep-space probes have lower navigational accuracy [3, 5, 8, Hence to date the effect could not be verified with another spacecraft.

The inability to explain the anomalous acceleration of the Pioneer spacecraft with conventional physics has contributed to the growing discussion about its origin. The possibility that it could come from a new physical effect is now being seriously considered. In particular the coincidence in magnitude of the Pioneer anomaly and the Hubble acceleration has stirred the suggestion that the Pioneer anomaly could be related to the cosmological expansion.

One of the obstacles for attempting an explanation of the Pioneer anomaly in terms of new physics is that a modification of gravity, large enough to explain the Pioneer anomaly, is likely to run into contradiction with the planetary ephemerides. This is readily illustrated by adding a term corresponding to the Pioneer anomaly to the Newtonian potential of the Sun,

$$
V(r)=-\frac{\mu_{\odot}}{r}-a^{*} r
$$

( $\mu_{\odot}$ is the reduced mass of the Sun, $r$ is the heliocentric distance, $a^{*} \approx 9 \times 10^{-10} \mathrm{~m} / \mathrm{s}^{2}$ is the anomalous acceleration) and considering the orbit of Neptune. At $30 \mathrm{AU}$ the Pioneer anomaly is visible in the Doppler data of both Pioneer 10 and 11 . The influence of an additional radial acceleration of $9 \times 10^{-10} \mathrm{~m} / \mathrm{s}^{2}$ on Neptune is conveniently parameterised by a change of the effective reduced Solar mass $\mu_{\odot}$, felt by the planet (cf. [21]). The value resulting for the anomaly, $\Delta \mu_{\odot}=a^{*} r_{\Psi}^{2} \approx 1.4 \times 10^{-4} \mu_{\odot}$, is nearly two orders of magnitude beyond the current observational constraint of $\Delta \mu_{\odot}=(-1.9 \pm 1.8) \times 10^{-6} \mu_{\odot}$ [22. Similarly the Pioneer 11 data contradict the Uranus ephemerides by more than one order of magnitude. Thus, the Pioneer anomaly can hardly be ascribed to a gravitational force since this would indicate a considerable violation of the weak equivalence principle. In particular, planetary constraints rule out an explanation in terms of a long-range Yukawa force [3, 23. Hence, more subtle explanations are to be attempted.

One line of reasoning is to consider an effect on the radio signal rather than a force on the spacecraft. Already the principle investigators have considered several phenomenological models of accelerating time 3. The main purpose of these models was to investigate the possibility of a systematic drift of atomic clocks. Most of the phenomenological models failed the cross-check with tracking data from other spacecraft. Only a time acceleration restricted to the signal propagation itself yielded a good fit to all spacecraft data although this model is still statistically disfavoured to a real deceleration of the spacecraft. The time acceleration of this model is indistinguishable from a run-time/travel-distance dependent blueshift of the radio signal.

To first order in $v / c$ the anomalous Doppler drift is related to the anomalous acceleration as

$$
\frac{1}{\nu} \frac{d \nu}{d t}=-\frac{a^{*}}{c}
$$

where $\nu$ is the emitter frequency of the signal, $v$ is the spacecraft velocity and $c$ is the velocity of light (cf. [3]). Note that $a^{*}$ is negative since it indicates a deceleration. At first sight this coincidence in phenomenology between an anomalous deceleration and an anomalous blueshift is surprising. It gets explained if one considers that the anomaly was only thoroughly investigated for the part of the Pioneer trajectories through the outer Solar system: Here the back-reaction of the spacecraft's orbit to a small perturbing force can be neglected and an anomalous acceleration can be treated linearly to high accuracy $[5]{ }^{1}$

${ }^{1}$ This simple observation illustrates the need for the analysis of the full Doppler data of Pioneer 10 and 11 because from data further inward in the Solar system a discrimination between a real force and a blueshift might be possible through the presence or absence of a change of the orbital parameters due to the anomaly. 
Several theoretical models have been put forward that implement an anomalous blueshift by very distinct mechanisms [24]-30]. The works [24, 25] consider the anomaly as a kinematical effect of the cosmological expansion. The anomaly arises from the fact that the coordinate system, in which local measurements are carried out, is not a synchronous one. The studies [26, 27] consider an adiabatic effect of the cosmic expansion on the phase of light viewed as the phase of a quantum state. Whereas [26] considers a closed path Berry phase, [27] drops the closed path requirement and considers an open path Berry phase. In [28-30] the anomaly arises from a time dependence of the local metric which leads to an effective time acceleration.

All of the above models to explain the blueshift of the Radio signals transponded by the Pioneers have to be considered as incomplete. This is most obvious for the model of [24, 25], where only a Robertson-Walker metric is considered and the influence of the gravitational field of the Sun is completely neglected. This seems too much of a simplification considering the predominant opinion that the local Schwarzschild geometry of the Solar system remains practically unaffected by the cosmological expansion (see the contribution of C. Lämmerzahl in this volume). The problem is ameliorated a bit for the quantum effect considered in [26, 27] because in this case one could argue that the adiabatic evolution of quantum states is governed by a different metric than the non-adiabatic dynamics of large bodies. Also the definition of the open-path Berry phase in 27] does not seem to be compatible with the general discussion of the open-path Berry phase in [31. In the models of [28-30] the embedding problem does not seem to spoil the model because both the cosmic and the local metric are treated as perturbations of a locally flat metric and can (at least formally) be superimposed linearly. However the model of 228, 29] suffers from the introduction of two ad-hoc coupling parameters between the electromagnetic and the gravitational field [32. Furthermore, the models [28]-30] lack a relativistic derivation of the background potential from the cosmological parameters. Despite of the deficiencies of the current models the idea that the Pioneer anomaly is caused by a blueshift of light is attractive because it automatically satisfies all constraints from planetary ephemerides.

\subsection{Relevance for LISA}

Among the proposed explanations of the Pioneer anomaly, most would have no significance for LISA. For example, this is the case for all models based on systematics generated onboard the Pioneer spacecraft. Generally, if the anomaly corresponds to a real acceleration on the Pioneers, the anomaly should have no influence on LISA. This can be concluded from the fact, that the LISA orbit is practically identical to the Earth's orbit. For the Earth itself an anomalous acceleration of the magnitude of the Pioneers would lead to an orbital perturbation which is beyond current observational limits (cf. [21]). Hence only a considerable violation of the weak equivalence principle (e.g. between bodies of different mass) could result in an anomalous acceleration on LISA but not on the Earth. On the other hand an anomalous blueshift of light could be highly relevant for LISA, since the mission is supposed to detect GWs through small frequency shifts. The blueshift for light travelling along an arm of LISA is found by integrating Eq. (2) in time,

$$
\frac{\Delta \nu^{*}}{\nu_{0}}=-\frac{a^{*}}{c} T
$$

where $T$ is the light travel time and $\nu_{0}$ is the laser frequency. For the LISA values, $\nu_{0} \approx 3 \times 10^{14} \mathrm{~Hz}$ and $T \approx 17 \mathrm{~s}$, one finds $\Delta \nu^{*} \simeq 1.5 \times 10^{-2} \mathrm{~Hz}$. Although the absolute blueshift is very small compared with the nominal frequency it might nevertheless be within the reach of LISA. Indeed, the corresponding relative change of the frequency is $\Delta \nu^{*} / \nu \simeq 10^{-16}$ and the expected value for the weakest GWs, that will be detectable by LISA, is about $10^{-23}$ [1]. The frequency shift due to the anomaly is therefore seven orders of magnitude bigger than the lowest signal to be detectable by LISA. The ability to measure the contribution of the anomalous blueshift will however depend on the sensitivity of LISA at the frequencies where the anomaly is present.

For a comprehensive analysis of the impact of the anomalous blueshift on LISA one has to take into consideration the change of the light travel time by passing GWs. In linear order in the GW strain $h$ 
the rate of change of the light travel time caused by a plane wave is proportional to the projection of the difference of the GW strains at the point of reception and the point of emission onto the light travel direction 33, 34,

$$
\frac{d}{d t} \Delta T=\frac{1}{2}(1+\beta)(h(t)-h[t+(1-\beta) T]) .
$$

Here $T$ is the unperturbed one-way light travel time, $\Delta T$ is the change of light travel time and $\beta$ is the cosine of the angle between the light travel direction and the normal of the wave front of the GW. The time $t$ is the time measured by a clock at the point of reception.

Writing Eq. (3) for the modified light travel time $T+\Delta T$ and using Eq. (4) to express $\Delta T$ by the linear term of a Taylor expansion, one obtains the frequency shift for the combined effect of the anomalous blueshift and GWs up to linear order in $a^{*}$ and $h$ for a one-way signal,

$$
\frac{\nu_{1}-\nu_{0}}{\nu_{0}}=-\frac{a^{*}}{c} T+\frac{1}{2}(1+\beta)\left[1-\frac{a^{*}}{c} T\right](h(t)-h[t+(1-\beta) T]),
$$

where $\nu_{1}$ is the frequency at reception. Eq. (5) is generic for any model of a homogeneous isotropic blueshift or time-acceleration. In particular it holds for the models considered in [3-Ranada:2004mf.

Depending on the model there might arise one subtlety, which has not been addressed up to now. In the same way as the electromagnetic waves are blueshifted an analogous blueshift might arise for the GWs. For example, this is the case in the models of [28]-[30], where the anomalous blueshift originates from a time-dependent term in the $g_{00}$ component of the metric caused by a homogeneous cosmological background potential. This additional term leads to a modified dispersion relation for all types of waves. The anomalous blueshift of GWs could be investigated by a parameter estimation of the dispersion relation via matched filtering of GW signals detected by LISA. The method would be analogous to the search for a graviton mass in GW signals (cf. [35, 36, 37]). In the present study we restrict ourselves to the possible manifestations of the Pioneer anomaly in electromagnetic waves because the occurrence of a blueshift of GWs is model dependent and hence would hardly allow a generic statement about the LISA's capability to verify the Pioneer anomaly.

Rather than the one-way response function of Eq. (5), the two-way response function of a signal transponded back to its emitter is the relevant observable for LISA. It is found analogous to Eq. (5) as

$$
\begin{aligned}
\frac{\nu_{2}-\nu_{0}}{\nu_{0}}=-\frac{a^{*}}{c} T+\frac{1}{2}(1+\beta)\left[\begin{array}{rl}
1 & \left.-\frac{a^{*}}{2 c} T\right]
\end{array}\right. & h(t) \\
& \quad-\beta\left[1-\frac{a^{*}}{2 c} T\right] h(t+(1-\beta) T / 2)-\frac{1}{2}(1-\beta)\left[1-\frac{a^{*}}{2 c} T\right] h(t+T),
\end{aligned}
$$

where $T$ now denotes the unperturbed two-way light time and $\nu_{2}$ is the frequency at reception [33, 34]. The anomalous blueshift contributes to the frequency shift by two types of terms. On the one hand it arises proportional to the unperturbed light travel time. If the light travel time is time dependent $T(t)$, as will be the case for LISA, the influence of the anomalous blueshift arises at the different frequencies contained in $T(t)$ and at null frequency anyway. On the other hand the anomalous frequency shift appears as a crossterm with the GW strain. This effect is hence suppressed by the smallness of the GW strain but still several orders of magnitude larger than terms quadratic in the GW strain. Both manifestations of the anomalous blueshift will be investigated in the following.

\section{Frequency domain analysis}

In the previous section, we have discussed how the Pioneer anomaly could find its explanation in a blueshift of light and we found the generic Doppler response function to a plane GW in the presence of an anomalous blueshift. This Doppler response function describes the influence of the anomalous blueshift on GW detection by an interferometer arm of LISA. In the following we analyse this signal both inside and 
outside the sensitivity band of the LISA. This is done first through an analysis in the frequency domain [38] and afterwards in the framework of TDI [11, 39.

The noise cancelation algorithm in the frequency domain 38, 40, is now considered as obsolete for the LISA data analysis and has been superseded by TDI, which achieves a far superior cancellation of laser phase noise in the signal than the frequency domain algorithm. For our purposes the analysis in the frequency domain has the considerable advantage that it allows an intuitive understanding of the influence of the anomalous blueshift. In this method the magnitude of various contributions to the signal can be easily compared and have a direct interpretation in term of phase shifts of an idealised signal.

This convenient interpretation is partially lost in TDI, in which combinations of signals are formed following an algebraic method in order to cancel the dominant noise source of the interferometer. A more physical picture can in some part be regained by interpreting the TDI combinations as synthesised interferometers. Nevertheless an investigation of the impact of an anomalous blueshift purely in terms of TDI might miss important effects of the blueshift, which might be cancelled by the specific symmetries of the TDI observables. On the other hand the possibilty exists that TDI combinations become particularly sensitive to the blueshift on behalf of their symmetries. Hence it is important to investigate if TDI remains unimpaired by an anomalous blueshift and if TDI is capable of detecting a potential anomaly.

Our analysis of the anomalous blueshift in the frequency domain is based on the method of [38. We amend the original discussion by the consideration of additional noise sources, such as acceleration noise, which were not addressed in [38] and we update the values of laser and shot noise to match the current expectations for LISA (cf. [1, 11]).

\subsection{The two-way Doppler signals}

For simplicity, we assumed in our analysis that each laser has the same fundamental frequency $\nu$, whereas in a realistic LISA configuration the frequency of the lasers may differ to each other by several hundreds of MHz. As a further simplification we start our discussion by assuming constant and exactly known (but unequal) lengths of the interferometer arms. This assumption will be dropped later.

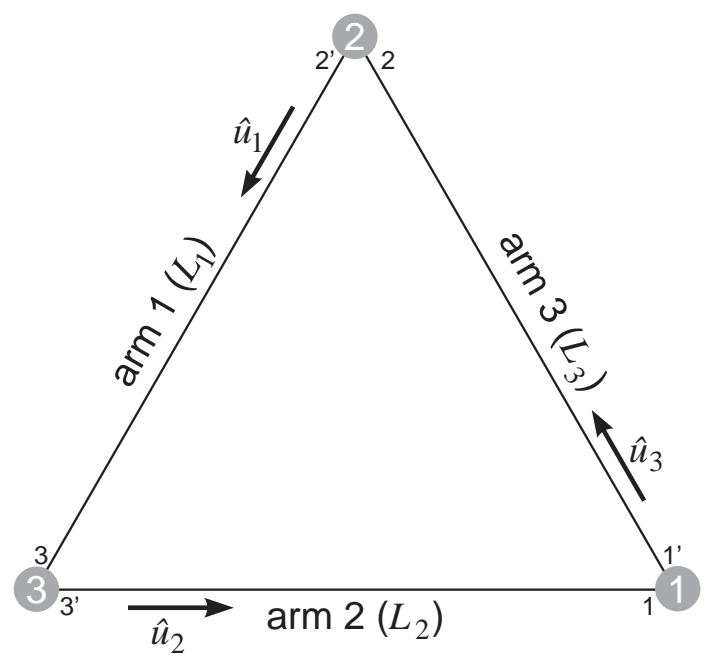

Figure 1: Geometry of the LISA formation.

The basic interferometer configuration is displayed in the Fig. 1. The distances between pairs of spacecraft are $L_{1}, L_{2}$ and $L_{3}$, with $L_{i}$ corresponding to the interferometer arm opposite to spacecraft $i$. The optical benches of each spacecraft are labelled by a number, which corresponds to that of the host spacecraft. An apostrophe allows to distinguish the two optical benches of the same spacecraft. In addition, a unit vector $\hat{u}_{i}$ is asigned to each arm, with $i$ being the label of the opposite spacecraft. The orientation of the three unit vectors are such that $\hat{u}_{1} L_{1}+\hat{u}_{2} L_{2}+\hat{u}_{3} L_{3}=0$. 
The phase of the signal received from a distant spacecraft of the LISA constellation is the sum of the following contributions:

1. The phase $2 \pi \nu l_{i}(t)$ due to the runtime of the signal, where $l_{i}(t)$ is the one-way light-time for the signal along the $i$ th interferometer arm. It changes due to the slow relative velocities between the spacecraft and on shorter timescales due to GWs.

2. The laser phase noise, $p_{i}(t)$, is the phase noise of the $i$ th laser, so that the phase of the $i$ th laser is $P_{i}=2 \pi \nu t+p_{i}(t)$.

3. The shot noise. Its effect is immediate at the time of reception, so that the response of the Doppler measurement at the $i$ th laser is simply given by $n_{i}(t)$.

4. The acceleration noise. The phase variation $\Delta \varphi$ of a signal depends on the path length $x$ through

$$
\Delta \varphi=\frac{2 \pi \nu}{c} \Delta x
$$

Therefore, the residual acceleration $\vec{a}_{i}(t)$ of the optical bench of the $i$ th spacecraft appears in the second derivative of the phase of the signal. Obviously, the residual accelerations at the two spacecraft, both at transmission and reception, have to be taken into account according to the following expression

$$
\Delta \varphi_{i}=\frac{2 \pi \nu}{c} \iint\left[\hat{u}_{j} \cdot \vec{a}_{i}(t)-\hat{u}_{j} \cdot \vec{a}_{k^{\prime}}\left(t-l_{j}\right)\right] d t^{2},
$$

where $i$ and $k^{\prime}$ are the end lasers of arm $j$ and $\Delta \varphi_{i}$ is the phase variation at the photodiode $i$. Note that this equation considers only the acceleration along the optical axis and does not take into account a possible turning of the optical bench.

5. The anomalous blueshift. Using Eq. (3) and defining $\alpha^{*} \equiv-a^{*} / c$ its contribution to the phase of the signal is given by

$$
\Delta \varphi_{i}^{*}(t)=2 \pi \alpha^{*} \int \Delta l_{i}(t) d t
$$

By taking into account all of the above contributions, the phase of the signal sent by the $k^{\prime}$ th laser and received at the $i$ th reads

$$
\begin{aligned}
\varphi_{i}(t)=2 \pi \nu\left(t-l_{j}(t)\right)+p_{k^{\prime}}\left(t-l_{j}\right)+ & n_{i}(t) \\
& +2 \frac{\pi \nu}{c} \iint\left[\hat{u}_{j} \cdot \vec{a}_{i}(t)-\hat{u}_{j} \cdot \vec{a}_{k^{\prime}}\left(t-l_{j}\right)\right] d t^{2}+2 \pi \alpha^{*} \int l_{j}(t) d t .
\end{aligned}
$$

At the reception, the incoming signal is beaten with the signal $P_{i}$ of the local laser to give the beat signal

$$
\sin \left(\varphi_{i}(t)\right)+\sin \left(P_{i}(t)\right)=2 \sin \left[\frac{\varphi_{i}+P_{i}}{2}\right] \cos \left[\frac{\varphi_{i}-P_{i}}{2}\right] .
$$

The high-frequency sine term is too fast to be read and is not used in the data analysis. Therefore, on the $j$ th arm, the phase of the beat signal read in the spacecraft photodiode is given by

$$
\begin{aligned}
s_{i}(t)= & \varphi_{i}(t)-P_{i}(t) \\
= & -2 \pi \nu l_{j}(t)+p_{k^{\prime}}\left(t-l_{j}\right)-p_{i}(t)+2 \pi \alpha^{*} \int l_{j}(t) d t+n_{k^{\prime}}(t) \\
& +2 \frac{\pi \nu}{c} \iint\left[\hat{u}_{j} \cdot \vec{a}_{i}(t)-\hat{u}_{j} \cdot \vec{a}_{k^{\prime}}\left(t-l_{j}\right)\right] d t^{2},
\end{aligned}
$$

where we have dropped the factor $1 / 2$ from the argument of the cosine in Eq. (11). Furthermore the two lasers on each spacecraft are tied to each other in phase by the exchange of a two-way reference signal between them. 
The two-way Doppler signal is then formed by the combination of the phase measurements from two photodiodes on the same arm (cf. [38]),

$$
\begin{aligned}
z_{i}(t)= & s_{i}(t)+s_{k^{\prime}}\left(t-l_{j}\right) \\
= & p_{i}\left(t-2 l_{j}\right)-p_{i}(t)-4 \pi \nu l_{j}(t)+4 \pi \alpha^{*} \int l_{j}(t) d t+n_{k^{\prime}}(t)+n_{i}\left(t-l_{j}\right) \\
& +2 \frac{\pi \nu}{c} \iint\left[\hat{u}_{j} \cdot \vec{a}_{i}(t)-2 \hat{u}_{j} \cdot \vec{a}_{k^{\prime}}\left(t-l_{j}\right)+\hat{u}_{j} \cdot \vec{a}_{i}\left(t-2 l_{j}\right)\right] d t^{2} .
\end{aligned}
$$

In order to obtain $z_{i}(t), s_{k^{\prime}}(t)$ is sent to the $i$ th laser to be beaten with $s_{i}(t)$. Here, the beat signal is filtered in order to preserve the GW contribution, i. e. by reading the cosine term in the expression of a beat (analogous to Eq. (11)).

\subsection{Inside the sensitivity band}

In Eq. (13), the light travel time as a function of reception time $l_{j}(t)$ includes both the orbital motion of the spacecraft and the GWs. We write explicitly the contribution of the GWs by now considering $l_{j}(t)$ in as the nominal arm length in the undisturbed spacetime and adding the disturbance by the GW as a separate term. Using the Doppler response function, Eq. (5), the effect of a GW, transverse to the LISA plane, i. e. $\beta \equiv 0$, and with appropriate polarisation, on the two-way Doppler signal is given by

$$
\frac{\Delta \nu}{\nu}=\frac{1}{2} \epsilon\left(1+\alpha^{*} l_{j}\right)\left[h(t)-h\left(t-2 l_{j}\right)\right],
$$

where $\Delta \nu$ is the difference between the frequency of the signal sent and received at the central spacecraft and $h$ is the GW strain amplitude. The $\epsilon$ can take any value between -1 and 1 , depending on the orientation of the arm with respect to the polarisation of the GW. Particularly, for an angle of $60^{\circ}$ between the arms of LISA, one can have $\epsilon=1$ for one arm and $\epsilon=-1 / 2$ for the other (cf. [1] p. 102 for the general expressions). The GW adds a contribution to the signal, Eq. (13),

$$
\Delta \varphi_{\mathrm{gw}}=\epsilon \pi \nu \int\left(1+\alpha^{*}\right)\left[h(t)-h\left(t-2 l_{j}\right)\right] d t .
$$

In order to estimate the importance of each term in Eq. (13) and (14), it is useful to compute the power spectral density of $z_{i}(t)$. To begin, we restrict our study to the sensitivity band of LISA, i. e. from $10^{-4} \mathrm{~Hz}$ to $1 \mathrm{~Hz}$. In a first estimate we can drop the two terms, $-4 \pi \nu l_{j}(t)+4 \pi \alpha^{*} \int l_{j}(t) d t$ because the orbital motion has little impact at the frequencies of the sensitivity band. To compute the power spectral density, we consider the Fourier transform of $z_{i}(t)$,

$$
\begin{aligned}
& z_{i}(f)=p_{i}(f)\left[e^{4 \pi i f l_{j}}-1\right]+n_{i}(f)\left[1+e^{2 \pi i f l_{j}}\right] \\
& \quad+\nu a_{i}(f) \frac{\left[e^{4 \pi i f l_{j}}+2 e^{2 \pi i f l_{j}}+1\right]}{2 \pi c f^{2}}+\epsilon \nu h(f) \frac{\left[e^{4 \pi i f l_{j}}-1\right]}{2 i f}+\epsilon \nu \alpha^{*} l_{j} h(f) \frac{\left[e^{4 \pi i f l_{j}}-1\right]}{2 i f},
\end{aligned}
$$

where we have assumed that the shot noise and acceleration spectra for both optical benches are the same, $n_{i}(f)=n_{k^{\prime}}(f)$ and $a_{i}(f)=a_{k^{\prime}}(f)$. Furthermore we have assumed the maximum value for the direction cosine between $\vec{a}_{i, k^{\prime}}$ and $\hat{u}_{j}$. The Fourier transform, Eq. (16), supposes that the observing time is infinite. In practice, LISA is expected to operate in data-taking intervals of $T \sim 10000 \mathrm{rms}$ and thus, Eq. (16) only gives an estimate of the true spectrum. We will return to the effect of finite observation time below.

From Eq. (16) it can be read off immediately that the effect of the anomalous blueshift would be

undetectable. The blueshift enters the spectrum folded with the GW strain $h$. Hence its effect will be 15 orders of magnitude below the GW signal. This corresponds to a spectral power at least 10 orders of magnitude below the secondary noises, shot noise and acceleration noise (cf. 11, 11] for the estimated noise spectra for LISA). Currently no procedure exists to cancel the shot noise in the LISA signal. Hence the anomalous blueshift would be overwhelmed by the secondary noises and remain unnoticed. 
This conclusion has however to be reconsidered taking into account that the data-taking periods of LISA are limited in length. This leads to the leakage of spectral power to other frequencies. In particular, the low-frequency terms neglected in the two-way signal $z_{i}(t)$, Eq. (13), have now to be addressed. For typical integration times, $T \sim 10000$ s (cf. [11]), the arm length rate of change is nearly constant. Its magnitude depends on the position of the spacecraft along its orbit [1]. The relative velocity, $\vec{v}$ can reach up to $13 \mathrm{~m} / \mathrm{s}^{2}$ In the approximation of constant relative velocity the Fourier transform of $l_{j}(t)=l_{j, 0}+\left(v_{j} / c\right) t$ is given by

$$
\begin{aligned}
\widetilde{F . T} \cdot\left[l_{j}(t)\right]=\int_{0}^{T}\left(l_{j, 0}+\frac{v_{j}}{c} t\right) & e^{2 \pi i f t} d t \\
& =\frac{v_{j}}{c} e^{i \pi T f} \frac{\pi T f \cos (\pi T f)-\sin (\pi T f)}{2 \pi^{2} i f^{2}}+\left[l_{j, 0}+\frac{v_{j}}{2 c} T\right] e^{i \pi T f} \frac{\sin (\pi T f)}{\pi f},
\end{aligned}
$$

where $c l_{j, 0} \approx 5 \times 10^{9} \mathrm{~m}$ is the initial light time between the spacecraft. In Eq. (17) the constant term due to the arm length, $l_{j, 0}$, is dominant. This term will remain present even after the application of the laser noise cancellation algorithm in the frequency domain (cf. 38]). Using Eq. (17]) the Fourier transform of the two-way Doppler signal becomes

$$
\begin{aligned}
& z_{i}(f)=p_{i}(f)\left[e^{4 \pi i f l_{j}}-1\right]+n_{i}(f)\left[1+e^{2 \pi i f l_{j}}\right]+ \\
& \nu a_{i}(f)\left[e^{4 \pi i f l_{j}}+2 e^{2 \pi i f l_{j}}+1\right]+4 \pi \nu l_{j}(f)-\frac{2 \alpha^{*}}{i f} l_{j}(f) .
\end{aligned}
$$

In this expression, we have dropped the contribution of GWs because above it was found irrelevant for the discussion of the blueshift (See [38] for a discussion of the GW signal in terms of the frequency domain algorithm). The contributions to the amplitude power spectrum corresponding to Eq. (18) are shown in Fig. 2. The signal of the anomalous blueshift is higher than the secondary noise sources but below the laser phase noise. The nominal term from $l_{j}$, i. e. the orbital motion is much higher than the laser noise. Hence this term would have to be removed by a preprocessing method before the laser noise cancellation algorithm could be applied (see below). The signal of the anomalous blueshift is below the laser phase noise but above the secondary noise sources.

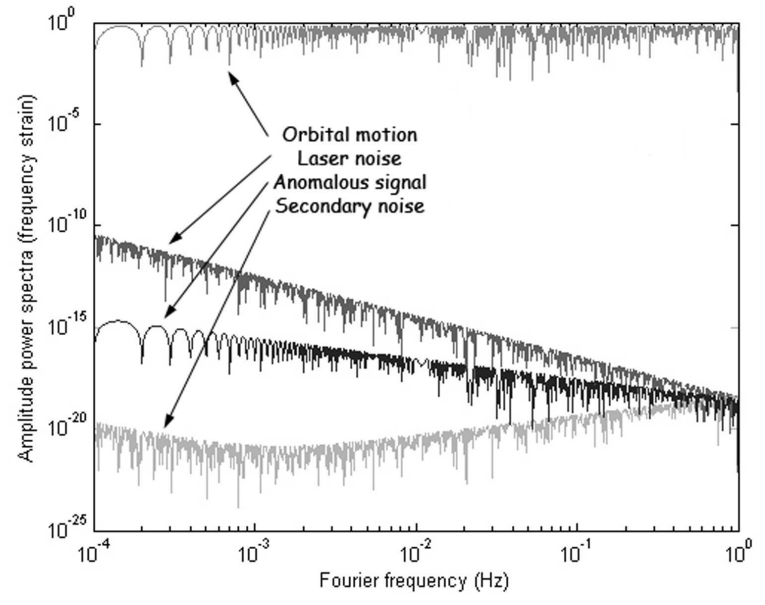

Figure 2: Amplitude power spectra contributing to the two-way Doppler signal in the sensitivity band.

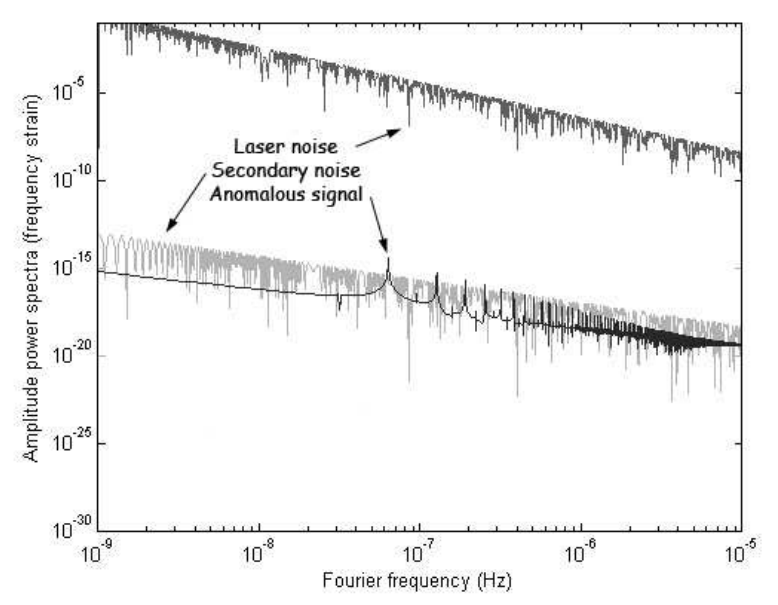

Figure 3: Amplitude power spectra contributing to the two-way Doppler signal outside the sensitivity band.

\footnotetext{
${ }^{2}$ See however [42] for recent suggestion of a modified orbit, which could reduce the relative velocity between the spacecraft by a factor of six.
} 
The further processing of the signal is distinct for the search for GWs and for the search for an anomalous blueshift. In the search for GWs the spectral leakage is unwanted. Hence a suitable approach is pre-multiplying the time-domain data sets by a window function before taking Fourier transform [11]. With this preprocessing, the laser noise cancellation can be performed and GWs could be detected. On the other hand, in the search for an anomalous blueshift the spectral leakage has to be preserved. However before the cancellation of the laser noise could be attempted, one has to generate a signal in which the laser noise is the dominant disturbance. Hence the nominal orbital term needs to be removed from the signal. The natural approach to this task would be to pre-process the data with information on the orbits acquired from a different source, e. g. ground tracking of the spacecraft.

The crucial question is how accurately we would need to determine the arm length of LISA to sufficiently remove the nominal orbital motion term. According to Fig. 2, a cancellation of about $10^{15}$ orders of magnitude would have to be performed. We suppose that the arm length $l_{j}$ is known up to a factor $k$, i. e. the real arm length $l_{j}$ differs from the assumed arm length $l_{j, \text { measured }}$ by the length $k l_{j}$, $l_{j, \text { measured }}=(1+k) l_{j}$. Then the Fourier transform of the one-way Doppler signal becomes

$$
\begin{aligned}
z_{i}(f)=p_{i}(f)\left[e^{4 \pi i f l_{j}}-1\right]+ & n_{i}(f)\left[1+e^{2 \pi i f l_{j}}\right] \\
& +a_{i}(f)\left[e^{4 \pi i f l_{j}}+2 e^{2 \pi i f l_{j}}+1\right]+4(1+k) \pi \nu l_{j}(f)-(1+k) \frac{2 \alpha^{*}}{i f} l_{j}(f) .
\end{aligned}
$$

Therefore, after the removal of the nominal orbital motion term from the knowledge of $l_{j \text {,measured, the }}$ term $4 k \pi \nu l_{j}(f)$ remains which has to be sufficiently low to detect the anomaly. However at $10^{-4} \mathrm{~Hz}$, this requirement corresponds to a knowledge of the arm length of about $10^{-15} \times l_{j}=5 \times 10^{-6} \mathrm{~m}$, which is far beyond the experimental capabilities of LISA.

In conclusion an anomalous blueshift of the magnitude of the Pioneer anomaly would remain undetected in the sensitivity band of LISA. Furthermore it would not affect LISA's capability to observe GWs. The blueshift remains unimportant because it is peaked around zero frequency and thus far away from the sensitivity band of LISA. However it is still worth considering the potential impact of the anomaly at frequencies below the sensitivity band of LISA where the effect becomes much larger.

\subsection{Outside the sensitivity band}

For long integration times, the arm-length rate of change cannot be treated as constant anymore. In the following we use the simplified analytical model of the LISA orbits described in [43, in which only the Kepler problem for each spacecraft is considered. Computing the corresponding power spectrum, one can plot the two-way Doppler signal, outside the sensitivity band of LISA.

Unfortunately, the noise spectra for LISA at frequencies below the measurement band have not yet been fully investigated (cf. 45]). For our purposes we use an extrapolation of the noise spectra obtained in [45. The acceleration noises might become considerably higher if a suspension mode for low frequencies would be implemented along the optical axes of the interferometer. However the results of [44 indicate that the best performance of LISA is obtained if the drag-free mode along the sensitivity axes is maintained also for low-frequencies. Hence an extrapolation of the noise spectra given in [45] should give a reasonable impression of the actual performance to expect from LISA at low frequencies.

The result is displayed in Fig. 3. The term due to the anomalous blueshift is of the same order of magnitude as the secondary noise but remains still below the laser phase noise. However, for the integration time required to reach these frequencies, the laser noise cancellation algorithm can no longer be implemented because the arm length changes from the orbital motion of LISA are so big that the algorithm becomes ineffective [38, 40]. Thus the presence of an anomalous blueshift cannot be revealed at low frequencies either.

To summarise, the anomalous blueshift would have an amplitude several orders of magnitude higher than the weakest GWs detectable, on the LISA's arms. However, this "large" impact of the anomaly comes from the constant part of the arm lengths. Hence it is located at null Fourier frequency while 
relevant GWs for LISA are expected at Fourier frequencies between $10^{-4}$ and $1 \mathrm{~Hz}$. In the sensitivity band of LISA, the effect of the anomaly is well below all the instrumental noises and hence is neither detectable nor does it have an impact on the GW detection. With a finite observation time, power of the constant contribution of the anomaly can leak in the sensitivity band. The analysis of the spectral leakage of the anomalous blueshift would however require a knowledge of the arm length that would have to be far more precise than it is achievable. Below the sensitivity band, we found that the contribution of the anomalous blueshift should be just above the secondary noise sources but still below the laser phase noise. On these timescales, the arm lengths change much more than it is allowed to remove efficiently the laser phase noise. Therefore, we can conclude that the Pioneer anomaly has no impact on the GW detection and cannot be detected with the frequency domain method.

\section{Time delay interferometry}

Time delay interferometry (TDI) is a noise cancellation method for unequal arm interferometers that is performed in the time domain [11, 39, 46, 47. (see also the contribution by M. Tinto in this volume). The basic principle of TDI consists in combining appropriate one-way Doppler signals in order to remove the laser phase noise (actually, it also cancels the acceleration noise of the optical benches). Whereas TDI had originally been developed as a purely algebraic method its data combinations have a physical interpretation as virtual measurements of a synthesised interferometer 48. The major question to be addressed here is how the Pioneer anomaly affects the TDI combinations. Since the frequency domain study showed that the effect of the anomalous blueshift is negligible when folded with the GW strain, we can restrict our attention on the anomalous blueshift occurring at low frequencies.

\subsection{Linear Data combinations}

In principle there is an unlimited number of TDI observables corresponding to more and more complicated synthesised interferometers. For applications to LISA the number of beams to combine is usually limited to eight in the limit of a static interferometer. For this maximum number of beams there are ten linear combinations, which cancel the laser noises from all the spacecraft.

These TDI combinations cancel the laser phase noise of an interferometer at rest with unequal but constant arm lengths and are commonly dubbed first generation TDI. For the nominal operation mode of LISA, the unequal-arm Michelson interferometer, three independent possible combinations exist which are called $X, Y$ and $Z$. In the following we only consider the $X$ combination,

$$
\begin{aligned}
X=y_{32,322}-y_{23,233}+ & y_{31,22}-y_{21,33}+y_{23,2}-y_{32,3}+y_{21}-y_{31} \\
& +\frac{1}{2}\left(-z_{21,2233}+z_{21,33}+z_{21,22}-z_{21}\right)+\frac{1}{2}\left(+z_{31,2233}-z_{31,33}-z_{31,22}+z_{31}\right) .
\end{aligned}
$$

The Doppler data to be analysed are now called $y_{i j}=\Delta \nu / \nu$, where $\Delta \nu$ is the frequency deviation from the centre frequency $\nu$. The subscripts label the transmitting and receiving spacecraft. The convention is that $y_{i j}$ is the beam transmitted from spacecraft $i$ and received at spacecraft $j$. Internal metrology signals to correct for optical bench motions are denoted by $z_{i j}$, with the same labelling convention. These will however play no role in our considerations because their travel times are too short to show an appreciable anomalous blueshift. They are hence omitted from now on. Delay of laser data streams is indicated by commas in the subscripts: $y_{31,23}=y_{31}\left(t-l_{2}-l_{3}\right)=y_{31,32}$, etc. $\left(l_{i}\right.$ is the light-time on the $i$-arm).

The $Y$ and $Z$ combinations are obtained from the $X$ combination by cyclic relabelling of the spacecraft. Hence our results hold for all three of the unequal-arm Michelson combinations. It is easy to verified by direct substitution of the laser noise contribution, that the combination, Eq. (20), does not contain any

laser noise. In the unequal-arm Michelson combinations, each one-way signal occurs twice, at two different times; one term is added and the other subtracted. As a consequence, the Pioneer anomaly component, given by Eq. (3),

$$
y_{i j}^{*}=-\frac{a^{*}}{c} l_{i}(t)=\alpha^{*} l_{i}(t)
$$


disappears. Even the spectral leakage of the data has no impact because the terms, which contain the anomaly are all cancelled exactly. The same property holds for the other combinations of the data present in the literature (see 39] for a description of the other combinations).

In the combinations called $\alpha, \beta, \gamma$ and $\zeta$, which represent synthetic Sagnac interferometers, the contribution of the anomaly would not be cancelled if the frequency shift would depend on the direction of the light beam with respect to the Sun. To obtain such a direction dependent anomaly, that does not decay significantly over tens of AUs, one would however have to resort to exotic ideas like a topological defect located in the Sun. Such a model is hard to envisage and no such effect has been suggested as an explanation of the Pioneer anomaly. Hence we do not further consider this possibility.

\subsection{Effect of the orbital motion on Time Delay Interferometry}

The first generation TDI observables, as presented above, have been formulated in the limit, that LISA is fixed in space. However, each year, LISA will accomplish a complete rotation around its centre and the symmetries provided by a fixed interferometer will be broken. Because of this loss of symmetry, the contribution of the anomalous blueshift, arising on each arm, would not be cancelled completely anymore in the TDI combinations. Moreover, the laser phase noise does not cancel exactly, either. More complicated TDI combinations have been developed to overcome this problem [49, 50, 51. In addition to the rotation, there occurs a flexing of the arms of the detector, which is caused by the orbital motion and the perturbations of the planets. The interaction of these two effects with the anomaly is considered in the following.

\subsubsection{The effects of rigid rotation}

For the discussion of rigid rotation, a more subtle notation for the Doppler signals is required because the light travel times will now depend on the direction of the signal with respect to the rotation. While in the previous section $L_{3}$ was the length of the arm between the first and the second spacecraft, we denote now by $L_{12}$ the length travelled by the signal sent from spacecraft 1 and received at spacecraft 2 . The length travelled by the signal sent from spacecraft 2 and received at spacecraft 1 is called $L_{21}$. As illustrated

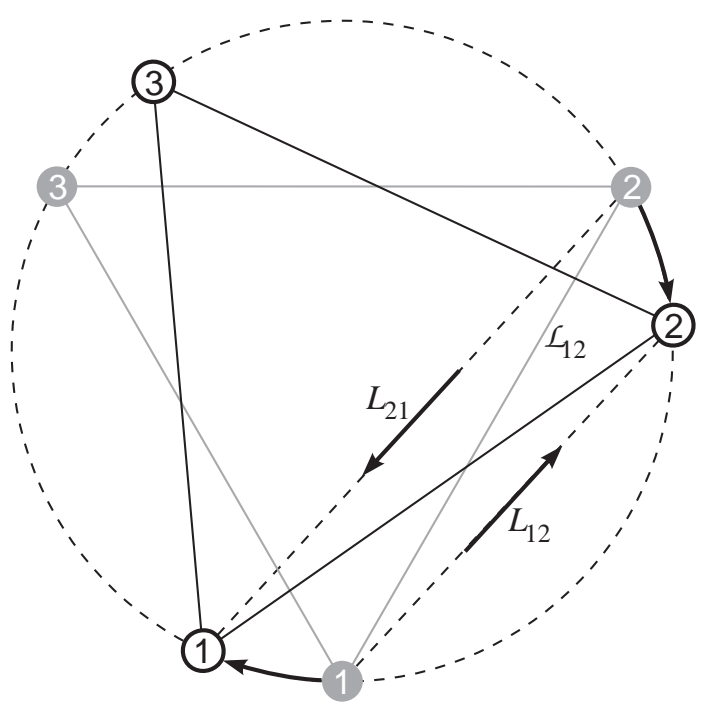

Figure 4: The rotation of the interferometer breaks the direction symmetry in the arm lengths.

in Fig. 4 the interferometer is rotating in the clockwise direction if viewed from the celestial pole. The spacecraft move while the signals are travelling along the arms. If we define the length of the arm between spacecraft 1 and 2 to be $\mathcal{L}_{12}$ in the limit of no rotation, then the actual distance travelled by the signal from spacecraft 1 to spacecraft 2 will be $L_{12}<\mathcal{L}_{12}$. In the same manner, the signal from spacecraft 2 to 
spacecraft 1 will have to reach spacecraft 1 in its motion and will therefore travel a distance $L_{21}>\mathcal{L}_{12}$. Hence also the magnitude of an anomalous blueshift on an arm would depend on the direction, in which the signal has travelled. Then, if the signals, which have travelled on the same arm but in opposite direction, are subtracted, a residual contribution of the anomalous blueshift would remain.

The direction dependence of the light travel times has different effects on the induvidual TDI combinations. For the unequal-arm length interferometric combinations $X(t), Y(t)$ and $Z(t)$, the contribution of the anomaly still cancels exactly. Indeed, if we take the $X(t)$ combination (the reasoning is the same for $Y(t)$ and $Z(t)$ ), we see that the one-way signals appear twice for a given direction with opposite signs in the combination but that they are delayed by different times. For a rigid rotation the relation $L_{i j}(t+\tau)=L_{i j}$ holds and the contribution of the anomaly is cancelled. The $(P, Q, R),(E, F, G)$ and $(U, V, W)$ have the same property so that one reaches the same conclusion for these combinations.

For the Sagnac combinations $(\alpha, \beta, \gamma$ and $\zeta)$, the structure of the signal is different (cf. 39]). In these observables two signals from each arm, running in opposite direction are combined. For instance the signal $\zeta$ reads,

$$
\zeta=y_{32}-y_{23,3}+y_{13,3}-y_{31,1}+y_{21,1}-y_{12,2} .
$$

Hence the effect of the anomaly is not totally removed. The anomalous component in Eq. (22) reads

$$
\zeta^{*}=\alpha^{*}\left(l_{12}-l_{13}+l_{23}-l_{21}+l_{31}-l_{32}\right) .
$$

The terms of this equation can be grouped into two parts: $\Delta l_{-} \equiv l_{12}+l_{23}+l_{31}$, which is the total time around the interferometer in the counterclockwise direction and $\Delta l_{+} \equiv l_{13}+l_{21}+l_{32}$, which is the total time in the clockwise direction. Even for a perfectly rigid triangle, the times of flight are not equal. Since the LISA constellation rotates in clockwise direction, we always have $\Delta l_{-}<\Delta l_{+}$. The corresponding Sagnac time shift is given by, cf. [49],

$$
\Delta l_{-}-\Delta l_{+}=\Delta l_{\text {Sagnac }}=\frac{4 A \Omega}{c^{2}} \approx \frac{2 \pi \sqrt{3} L^{2}}{c^{2} T} .
$$

Here $\Omega$ is the angular velocity of the rotation, $A$ is the area enclosed by the light path, $T$ is the period of rotation and $L$ is a typical arm length. For the LISA orbit $\left(T=1\right.$ year and $\left.L=5 \times 10^{9} \mathrm{~m}\right)$, the Sagnac effect has the magnitude $\Delta l_{\text {Sagnac }}=-10^{-4} \mathrm{~s}$. Therefore, the residual effect of the anomalous blueshift on the combination $\zeta$ would be

$$
\zeta^{*}=\alpha^{*}\left(\Delta l_{-}-\Delta l_{+}\right) \simeq 3 \times 10^{-22} .
$$

The same result is obtained for the other Sagnac combinations $\alpha, \beta, \gamma$. The effect of the anomalous blueshift is to add a constant frequency shift in the Sagnac combinations. The amplitude of this additional Doppler shift would be comparable to the weakest GWs detectable by LISA. However, the optimal sensitivity of LISA occurs in a Fourier frequency range far from the zero Fourier frequency, where the constant residual contribution of the anomaly has its impact. Hence, again the effect would not be detectable.

As mentioned above the rigid rotation induces a Sagnac effect on the noises as well. In order to maintain noise cancellation up to linear order in the rotational velocity for all observables modified TDI has been introduced, in which each Doppler signal from a specific arm enters twice travelling in the same direction. In modified TDI the anomalous blueshift is cancelled at linear order in the rotational velocity and the effect of the anomaly would become even smaller.

\subsubsection{The effects of flexing}

As we have seen in Section 3.3 the arm lengths of LISA don't remain constant due to the orbital motion and the perturbations of the orbits by the planets. Unlike the rigid rotation, the flexing of the arms does not preserve the continuous symmetry, $L_{i j}(t+\tau)=L_{i j}(t)$. For example, the $X$ combination, Eq. (20), 
becomes for time varying runtimes (cf. [49])

$$
\begin{aligned}
X= & y_{12}\left[t-l_{31}-l_{13}^{(1)}-l_{21}^{(2)}\right]-y_{13}\left[t-l_{21}-l_{12}^{(1)}-l_{31}^{(2)}\right]+y_{21}\left[t-l_{31}-l_{13}^{(1)}\right] \\
& -y_{31}\left[t-l_{21}-l_{12}^{(1)}\right]+y_{13}\left(t-l_{31}\right)-y_{12}\left(t-l_{21}\right)+y_{31}(t)-y_{21}(t)
\end{aligned}
$$

where $l_{21} \equiv l_{21}(\mathrm{t}), l_{31} \equiv l_{31}(\mathrm{t}), l_{12}^{(1)} \equiv l_{12}\left(t-l_{21}\right), l_{13}^{(1)} \equiv l_{13}\left(t-l_{31}\right), l_{21}^{(2)} \equiv l_{21}\left(t-l_{31}-l_{13}^{(1)}\right)$ and $l_{31}^{(2)} \equiv l_{31}\left(t-l_{21}-l_{12}^{(1)}\right)$. Using Eq. (21), the contribution of the anomalous blueshift in the $X$ combination is given by

$$
\begin{aligned}
X^{*}= & \alpha^{*}\left[l_{12}\left(t-l_{31}-l_{13}^{(1)}-l_{21}^{(2)}\right)-l_{13}\left(t-l_{21}-l_{12}^{(1)}-l_{31}^{(2)}\right)+l_{21}\left(t-l_{31}-l_{13}^{(1)}\right)\right] \\
& +\alpha^{*}\left[-l_{31}\left(t-l_{21}-l_{12}^{(1)}\right)+l_{13}\left(t-l_{31}\right)-l_{12}\left(t-l_{21}\right)+l_{31}(t)-l_{21}(t)\right] \\
= & \alpha^{*}\left[l_{12}^{(3)}-l_{13}^{(3)}+l_{21}^{(2)}-l_{31}^{(2)}+l_{13}^{(1)}-l_{12}^{(1)}+l_{31}-l_{21}\right],
\end{aligned}
$$

where $l_{12}^{(3)} \equiv l_{12}\left(t-l_{31}-l_{13}^{(1)}-l_{21}^{(2)}\right)$ and $l_{13}^{(3)} \equiv l_{13}\left(t-l_{21}-l_{12}^{(1)}-l_{31}^{(2)}\right)$. The arm lengths can be estimated by their first order changes $l_{i j}^{(n)}=l_{i j}(t)-n V_{i j} l$, where $V_{i j}$ is the rate of change of the arm's light travel time in seconds per second and $l$ is a typical one-way light-time [49. Then we find from Eq. (27)

$$
X^{*}=4 \alpha^{*}\left(V_{13}-V_{12}\right) l=6 \times 10^{-24},
$$

where, in accordance with [4], we have used $V_{13}-V_{12} \lesssim 10(\mathrm{~m} / \mathrm{s}) / c$. This relation determines the maximum effect of the blueshift due to the flexing induced by the orbital motion. The Doppler shift stays below the optimal sensitivity of LISA which is about $10^{-23}$. Results at the same order of magnitude are obtained for all TDI combinations. Hence we conclude that the effect of the anomalous blueshift due the flexing of the interferometer arms will not be detectable.

The flexing of the arms inhibits laser noise cancellation to first order in the velocities for first generation TDI or modified TDI. To achieve the noise cancellation up to first order, inclusive, another set of observables has been designed. The so-called second generation TDI achieves the cancellation by having not only a signal for each arm entering twice in the same direction but also having each term linear in the change rate of the length of an arm entering twice. Hence second generation TDI also cancels the anomalous blueshift from terms linear in the change rate of the arms.

We found that TDI observables, especially those of second generation TDI, are particularly insensitive to the anomalous blueshift. This result has a simple geometric justification. The anomalous blueshift of sizable magnitude arises proportional to arm-length differences. However TDI is based on combining signals in a way, that yields overall light-times of zero (cf. [48]). Hence the very principle of the TDI algorithm leads to an automatic cancellation of the anomalous blueshift in the signal.

\section{$5 \quad$ Summary and conclusions}

The Pioneer anomaly is attracting a growing interest in the scientific community. Hence a verification of the effect beyond the Pioneer data would be highly desirable. Here we studied in which way the LISA mission could contribute to a test of the Pioneer anomaly. Due to its Earth-like orbit LISA would most likely not experience an anomalous force since this would require a strong violation of the weak equivalence principle. On the other hand, if one interprets the Pioneer anomaly as an anomalous blueshift of light this effect would affect also the LISA interferometer.

Several models in the literature consider the Pioneer anomaly as a homogeneous and isotropic blueshift originating from the cosmic expansion through various mechanisms 24]-30. All of these distinct models lead to a common Doppler response function for LISA up to linear order in the anomalous blueshift and in the GW strain. We derived this Doppler response function as an extension of the well known two-point 
response to GWs. We found that the blueshift arises on the one hand as a cross term with the GW signal and on the other hand as a low-frequency bias depending on the interferometer arm length. The cross term with the GW signal is much larger than a possible second-order GW term but still too small to be detectable by LISA.

The low-frequency term was found to induce a relative frequency shift of $10^{-16}$, which is several orders of magnitude larger than the weakest measurable GW strain of $10^{-23}$. The implications of this number are however not immediate because the anomalous blueshift arises at zero frequency whereas the LISA sensitivity lies between $10^{-4} \mathrm{~Hz}$ and $1 \mathrm{~Hz}$.

Consequently we investigated the power spectral density of the anomalous frequency shift, that arises from the orbital motion of the LISA satellites. We considered both, short times and timescales, which comprise a considerable fraction of the orbital period of LISA. These results were compared with the noise spectra of LISA. Unfortunately, due to its low-frequency nature the anomalous blueshift is always overwhelmed by some noise source of the LISA interferometer. Hence an anomalous blueshift would remain undetectable.

This conclusion is then reconsidered in the framework of TDI, the current baseline method for laser phase noise cancellation in LISA. The dedicated discussion of TDI is necessary because the complicated signals synthesised in TDI could produce a by-chance amplification of a homogeneous isotropic signal. Our results show on the contrary that TDI cancels the blueshift in all data combinations to a high degree due to the inherent symmetries of the TDI observables. Only from the rotation and flexion of the interferometer, a residual contribution of the anomaly would arise. This effect would however be below the detection threshold of LISA. Hence an anomalous blueshift will not be recognisable in TDI and will not degrade the performance of TDI for the detection of GWs either.

In the present study we have focused our attention on models of the anomaly, that predict a homogeneous and isotropic blueshift. At first glance this seems a bit restrictive, since also models, in which the Pioneer anomaly arises from a central force, can lead to a considerable blueshift of light. An example is the model of [52], which introduces separate momentum-dependent gravitational couplings for the scalar and the conformal sector of the Einstein equations. In this model about half of the Pioneer anomaly is due an anomalous blueshift. However the blueshift induced into the LISA signal by a central force would be proportional to the difference in light-travel time between the way back and forth in a two-way signal, whereas a homogeneous and isotropic blueshift is proportional to the two-way light travel time. Thus the blueshift from a central force, that is supposed to explain the Pioneer anomaly, would in general have much less effect on the LISA signal than a homogeneous and isotropic blueshift.

In conclusion, LISA cannot be used to test the Pioneer anomaly and one will have to look for other options to verify if the Pioneer anomaly could be a novel physical effect. Considering the blueshift interpretation of the anomaly, missions for a test of general relativity by interferometry like LATOR 53. (see also the contribution by S. Turyshev in this volume) or ASTROD [54] (see also the contribution by Wei-Tou Ni in this volume) might be sensitive to this effect. However also these missions would face the problem that the anomalous blueshift becomes significant only at low frequencies, i. e. for large changes of the light travel time in the interferometer.

More promising - and probably mandatory if the Pioneer anomaly represents a force and not a blueshift - would be a test in the outer Solar system by radio-tracking of a deep space vehicle with very well know onboard systematics [7. Preferably this would be a dedicated mission to explore the anomaly although a planetary exploration spacecraft, which has been designed with the secondary goal to test the Pioneer anomaly could already gain considerable insights [5]. ${ }^{3}$ The analysis of the full archive of Pioneer 10 and 11 Doppler data, that is currently being initiated, might further help to identify mission scenarios that are especially suited for a test of the anomaly.

\footnotetext{
${ }^{3}$ See [55] 56] for an example implementation of a Pioneer anomaly test on a Pluto exploration mission.
} 


\section{Acknowledgements}

The authors would like to thank the organisers of the 359th WE-Heraeus-Seminar "Lasers, Clocks and Drag-Free: Key Technologies for Future High Precision Test of General Relativity" for the realisation of this stimulating conference. The authors acknowledge support by the Advanced Concepts Team of the European Space Agency where the major part of this work was carried out. This work has much benefitted for discussions with O. Jennrich and D. Izzo.

\section{References}

[1] K. Danzmann et al. [LISA Study Team], Laser Interferometer Space Antenna: A cornerstone mission for the observation of Gravitational waves, System and technology study report, ESA-SCI(2000)11, July 2000.

[2] J. D. Anderson, P. A. Laing, E. L. Lau, A. S. Liu, M. M. Nieto and S. G. Turyshev, "Indication, from Pioneer 10/11, Galileo, and Ulysses data, of an apparent anomalous, weak, long-range acceleration," Phys. Rev. Lett. 81 (1998) 2858 arXiv:gr-qc/9808081.

[3] J. D. Anderson, P. A. Laing, E. L. Lau, A. S. Liu, M. M. Nieto and S. G. Turyshev, "Study of the anomalous acceleration of Pioneer 10 and 11," Phys. Rev. D 65 (2002) 082004 arXiv:gr-qc/0104064.

[4] O. Bertolami and J. Paramos, "The Pioneer anomaly in a bimetric theory of gravity on the brane," Class. Quant. Grav. 21 (2004) 3309 arXiv:gr-qc/0310101.

[5] D. Izzo and A. Rathke, "Options for a non-dedicated test of the Pioneer anomaly," arXiv:astro-ph/0504634

[6] R. H. Sanders, "A tensor-vector-scalar framework for modified dynamics and cosmic dark matter," arXiv:astro-ph/0502222

[7] H. Dittus et al. [Pioneer Collaboration], "A mission to explore the Pioneer anomaly," to appear in the proceedings of the 39th ESLAB Symposium: Trends in Space Science and Cosmic Vision 2020, Noordwijk, The Netherlands, 19-21 Apr 2005, arXiv:gr-qc/0506139.

[8] M. M. Nieto and S. G. Turyshev, "Finding the origin of the Pioneer anomaly," Class. Quant. Grav. 21 (2004) 4005 arXiv:gr-qc/0308017.

[9] L. K. Scheffer, "Conventional forces can explain the anomalous acceleration of Pioneer 10," Phys. Rev. D 67 (2003) 084021 arXiv:gr-qc/0107092.

[10] J. L. Rosales, "LISA mission and the Pioneer anomaly", proposal in response to the Call for Themes for Cosmic Vision 2015-2025 of the European Space Agency (May 2004).

[11] M. Tinto and J. W. Armstrong, "Cancellation of laser noise in an unequal-arm interferometer detector of gravitational radiation," Phys. Rev. D 59 (1999) 102003.

[12] L. Lasher and J. Dyer, "Pioneer Missions," in: P. Murdin (ed.), Encyclopedia of Astronomy and Astrophysics (Institute of Physics Publishing, Bristol, UK, 2002), http://eaa.iop.org/abstract/0333750888/2187

[13] G. W. Null, "Gravity field of Jupiter and its satellites from Pioneer 10 and Pioneer 11 tracking data," AJ 81 (1976) 1153.

[14] M. M. Nieto and J. D. Anderson, "Using early data to illuminate the Pioneer anomaly," arXiv:gr-qc/0507052. 
[15] C. B. Markwardt, "Independent confirmation of the Pioneer 10 anomalous acceleration," arXiv:gr-qc/0208046.

[16] J. I. Katz, "Comment on "Indication, from Pioneer 10/11, Galileo, and Ulysses data, of an apparent anomalous, weak, long-range acceleration"," Phys. Rev. Lett. 83 (1999) 1892 arXiv:gr-qc/9809070.

[17] E. M. Murphy, "A prosaic explanation for the anomalous accelerations seen in distant spacecraft," Phys. Rev. Lett. 83 (1999) 1890 arXiv:gr-qc/9810015.

[18] J. D. Anderson, P. A. Laing, E. L. Lau, M. M. Nieto and S. G. Turyshev, "The search for a standard explanation of the Pioneer anomaly," Mod. Phys. Lett. A 17 (2002) 875 arXiv:gr-qc/0107022.

[19] B. Mashhoon, "Modification of the Doppler effect due to the helicity-rotation coupling," Phys. Lett. A 306 (2002) 66 arXiv:gr-qc/0209079.

[20] J. D. Anderson and B. Mashhoon, "Pioneer anomaly and the helicity-rotation coupling," Phys. Lett. A 315 (2003) 199 arXiv:gr-qc/0306024.

[21] C. Talmadge, J. P. Berthias, R. W. Hellings and E. M. Standish, "Model independent constraints on possible modifications of Newtonian gravity," Phys. Rev. Lett. 61, 1159 (1988).

[22] J. D. Anderson, E. L. Lau, T. P. Krisher, D. A. Dicus, D. C. Rosenbaum and V. L. Teplitz, "Improved bounds on nonluminous matter in Solar orbit," Astrophys. J. 448 (1998) 885 arXiv:hep-ph/9503368.

[23] S. Reynaud and M. T. Jaekel, "Testing the Newton law at long distances," Int. J. Mod. Phys. A 20 (2005) 2294 arXiv:gr-qc/0501038.

[24] J. L. Rosales and J. L. Sanchez-Gomez, "The 'Pioneer effect' as a manifestation of the cosmic expansion in the solar system," arXiv:gr-qc/9810085.

[25] J. L. Rosales, "The Pioneer effect: a cosmological Foucault's experiment," arXiv:gr-qc/0212019

[26] J. L. Rosales, "The Pioneer's anomalous Doppler drift as a Berry phase," arXiv:gr-qc/0401014.

[27] J. L. Rosales, "The Pioneer anomaly: The measure of a topological phase defect of light in cosmology," arXiv:quant-ph/0501041

[28] A. F. Ranada, "The Pioneer riddle, the quantum vacuum and the acceleration of light," Europhys. Lett. 63 (2003) 653 arXiv:gr-qc/0211052.

[29] A. F. Ranada, "The light speed and the interplay of the quantum vacuum, the gravitation of all the universe and the fourth Heisenberg relation," Int. J. Mod. Phys. D 12 (2003) 1755.

[30] A. F. Ranada, "The Pioneer anomaly as acceleration of the clocks," Found. Phys. 34 (2005) 1955 arXiv:gr-qc/0410084.

[31] A. K. Pati, "Adiabatic Berry phase and Hannay angle for open paths," Annals Phys. 270 (1998) 178 arXiv:quant-ph/9804057.

[32] N. Jafari and A. Shariati, "Comments on the quantum vacuum and the light acceleration," arXiv:gr-qc/0409113

[33] F. B. Estabrook and H. Wahlquist, "Response of Doppler spacecraft tracking to gravitational radiation," Gen. Rel. Grav. 6 (1975) 439.

[34] R. W. Hellings, "Spacecraft Doppler gravity wave detection. 1. Theory," Phys. Rev. D 23, 832 (1981).

[35] C. M. Will, "Bounding the mass of the graviton using gravitational-wave observations of inspiralling compact binaries," Phys. Rev. D 57 (1998) 2061 arXiv:gr-qc/9709011. 
[36] C. M. Will and N. Yunes, "Testing alternative theories of gravity using LISA," Class. Quant. Grav. 21 (2004) 4367 arXiv:gr-qc/0403100.

[37] E. Berti, A. Buonanno and C. M. Will, "Estimating spinning binary parameters and testing alternative theories of gravity with LISA," Phys. Rev. D 71 (2005) 084025 arXiv:gr-qc/0411129.

[38] Giacomo Giampieri, Ronald W. Hellings, Massimo Tinto, James E. Faller, "Algorithms for unequalarm Michelson interferometers," Optics Communications 123 (1996) 669.

[39] Sanjeev V. Dhurandhar and Massimo Tinto, "Time-delay interferometry," Living Rev. Relativity 8, (2005), 4. URL (cited on 15 August 2005): http://www.livingreviews.org/lrr-2005-4 arXiv:gr-qc/0409034.

[40] G. Giampieri, R. W. Hellings, L. Maleki, M. Tinto, K. Danzmann, J. Hough, D. Robertson, "Heterodyne laser tracking at high Doppler rates", Optics Communications 124 (1996) 313.

[41] W. M. Folkner, T. H. Sweetser, M. A. Vincent, F. Hechler and P. L. Bender, "LISA orbit selection and stability," Class. Quant. Grav. 14 (1997) 1405.

[42] K. R. Nayak, S. Koshti, S. V. Dhurandhar and J. Y. Vinet, "Reducing the flexing of the arms of LISA," arXiv:gr-qc/0507105.

[43] S. V. Dhurandhar, K. Rajesh Nayak, S. Koshti and J. Y. Vinet, "Fundamentals of the LISA stable flight formation," Class. Quant. Grav. 22 (2005) 481 arXiv:gr-qc/0410093.

[44] A. Hammesfahr et al., LISA, Study of the Laser Interferometer Space Antenna, final technical report, ESA Industrial Study at Phase A, ESTEC Contract no. 13631/99/NL/MS, DSS Report No. LI-RPDS-009 (Dornier Satellitensysteme GmbH, Friedrichshafen, Germany).

[45] P. L. Bender, "LISA sensitivity below 0.1 mHz," Class. Quant. Grav. 20 (2003) S301.

[46] F. B. Estabrook, M. Tinto and J. W. Armstrong, "Time delay analysis of LISA gravitational wave data: Elimination of spacecraft motion effects," Phys. Rev. D 62 (2000) 042002.

[47] M. Tinto, F. B. Estabrook and J. W. Armstrong, "Time-delay interferometry for LISA," Phys. Rev. D 65 (2002) 082003.

[48] M. Vallisneri, "Geometric time delay interferometry," arXiv:gr-qc/0504145.

[49] N. J. Cornish and R. W. Hellings, "The effects of orbital motion on LISA time delay interferometry," Class. Quant. Grav. 20 (2003) 4851 arXiv:gr-qc/0306096.

[50] D. A. Shaddock, M. Tinto, F. B. Estabrook and J. W. Armstrong, "Data combinations accounting for LISA spacecraft motion,” Phys. Rev. D 68 (2003) 061303 arXiv:gr-qc/0307080.

[51] M. Tinto, F. B. Estabrook and J. W. Armstrong, "Time delay interferometry with moving spacecraft arrays," Phys. Rev. D 69 (2004) 082001 arXiv:gr-qc/0310017.

[52] M. T. Jaekel and S. Reynaud, "Post-Einsteinian tests of linearized gravitation," Class. Quant. Grav. 22 (2005) 2135 arXiv:gr-qc/0502007.

[53] S. G. Turyshev et al. [LATOR Collaboration], "Fundamental physics with the laser astrometric test of relativity," to appear in the proceedings of the 39th ESLAB Symposium: Trends in Space Science and Cosmic Vision 2020, Noordwijk, The Netherlands, 19-21 Apr 2005, arXiv:gr-qc/0506104.

[54] W. T. Ni, “ASTROD: An overview," Int. J. Mod. Phys. D 11 (2002) 947. 
[55] A. Rathke, "Testing for the Pioneer anomaly on a Pluto exploration mission," in: A. Morseli, P. Picozza and M. Ricci (eds.), Proceedings of the third international workshop on Frontier Science 2004. Physics and Astrophysics in Space (INFN, Frascati, Italy 2004) arXiv:astro-ph/0409373.

[56] T. Bondo, R. Walker, A. Willig, A. Rathke, D. Izzo and M. Ayre, "Preliminary Design of an Advanced Mission to Pluto". to appear in the proceedings of the 24th International Symposium on Space Technology and Science, Miyazaki, Japan, June 2004, http://www.esa.int/gsp/ACT/doc/ACT-RPR-4200-ISTS2004.pdf. 